[Cierre dle edición 12 de noviembre del 2010]

\title{
El talento en niños y niñas de I Ciclo de la Educación General Básica: la perspectiva docente vrs la realidad educativa
}

\section{Talent in first, second, and third graders: teachers' perspectives vs. educational reality}

\author{
Diana Castro Rodríguez, \\ Escuela Neftalí Villalobos Gutiérrez \\ Heredia, Costa Rica \\ Zulay Pereira Pérez ${ }^{2}$ \\ División de Educación Básica del Centro de Investigación y Docencia en Educación (CIDE) \\ Universidad Nacional \\ Heredia, Costa Rica \\ Jéssica Solís Campos ${ }^{3}$ \\ Taller Pedagógico Montebello \\ Heredia, Costa Rica \\ Kenneth Ureña Rodríguez ${ }^{4}$ \\ Universidad Metropolitana Castro Carazo \\ San José, Costa Rica \\ Belbeth Villalobos Obando \\ Escuela Quince de Agosto \\ San José, Costa Rica
}

Recibido 14 de agosto de 2009• Aceptado 02 de diciembre de 2009

1 Licenciada en Pedagogía con énfasis en I y II Ciclos de la Educación General Básica de la Universidad Nacional, Costa Rica. Experiencia como docente en instituciones privadas y públicas del Sistema Educativo Costarricense. Ha atendido en su labor docente a niños y niñas con necesidades educativas especiales (problemas de conducta, déficit atencional, retraso mental, síndrome de Down, parálisis cerebral y síndrome de Asperger, aplicando con ellos y ellas adecuaciones curriculares de acceso, no significativas y significativas). Capacitaciones en evaluación, adecuaciones curriculares, necesidades educativas especiales, Comité de Apoyo, Feria Científica, entre otras. Actualmente labora en la Escuela Neftalí Villalobos Gutiérrez, Heredia, Costa Rica. Correo electrónico: casedorz.13@hotmail.com

2 Doctora en Educación, de la Universidad Estatal a Distancia, Costa Rica. Catedrática de la Universidad Nacional, Costa Rica. Máster y Licenciada en Psicología, de la Universidad de Costa Rica. Adscrita al Colegio Profesional de Psicólogos de Costa Rica. Ex coordinadora del Programa de Investigación en Epistemología Genética y Educación del IIMEC, actualmente el Instituto de Investigación en Educación (INIE) de la Universidad de Costa Rica (UCR). Ex Directora y docente de la División de Educación Básica del Centro de Investigación y Docencia en Educación de la Universidad Nacional, Costa Rica. Investigadora del Centro de Investigación y Docencia en Educación de la Universidad Nacional, Costa Rica. Tiene publicaciones en el campo de la Psicología y la Pedagogía. Correo electrónico: zpereira@una.ac.cr

3 Licenciada en Pedagogía con énfasis en I y II Ciclos de la Educación General Básica. Manejo de lenguajes computacionales y Lesco Tiene capacitación en Déficit Atencional y dificultades de aprendizaje. Posee seis años de experiencia como docente de primaria en instituciones del sistema educativo costarricense. Actualmente labora en el Taller Pedagógico Montebello, en la Provincia de Heredia. Actualmente es estudiante de la Universidad Nacional cursando el programa de inglés conversacional. yecasc09@hotmail.com

4 Máster en Administración Educativa. Licenciado en Pedagogía con énfasis en I y II Ciclos de la Educación General Básica. Profesor en la Universidad Metropolitana Castro Carazo, Costa Rica. Docente de Educación General Básica en instituciones privadas y públicas de Costa Rica. Profesor de francés en niveles de educación secundaria. Capacitación Proyecto SNIPE, España. Formación en alcoholismo y farmacodependencia, dibujo técnico, procedimientos geométricos y recursos tecnológicos en Educación. Actualmente labora en la Corporación Educativa Santa María, Costa Rica y en la Universidad Metropolitana Castro Carazo, Costa Rica. quinonis@yahoo.es kennethu@costarricense.cr

5 Licenciada en Pedagogía con énfasis en I y II Ciclos de la Educación General Básica. Experiencia docente en instituciones educativas costarricenses. Ha sido recuperadora del Programa de Escuelas para el Mejoramiento de la Calidad de la Educación y Vida en las Comunidades Urbanas de Atención Prioritaria, en el Comité de Apoyo Educativo y en el Comité de Evaluación. Ha trabajado con niños y niñas con necesidades educativas especiales y ha recibido capacitaciones en esta área. Formación en alcoholismo y farmacodependencia. Actualmente labora como docente en un Centro Educativo de Atención Prioritaria, la Escuela Quince de Agosto en San José. Correo electrónico: belbita-@hotmail.com 
Resumen. El artículo aborda el tema del talento. Destaca distintas concepciones sobre éste y plantea un acercamiento a la temática desde la visión de un talento en cada niño y niña. Presenta resultados de una investigación realizada con niños y niñas de I Ciclo de la Educación General Básica, en instituciones públicas y privadas del sistema educativo costarricense. Mediante el recurso del cuestionario y la observación de las dinámicas de aula, contrasta los planteamientos de los docentes y las docentes acerca del talento de sus estudiantes, con la realidad que se presenta en sus aulas. Señala el abismo entre las concepciones docentes y el escenario de sus prácticas profesionales.

Palabras clave. Niños y niñas de I Ciclo, talento, prácticas docentes, realidad educativa, creencias docentes.

Abstract. The article addresses the subject of talent by emphasizing different conceptions about talent and by proposing an approach to this theme, from the point of view of talent in boys and girls. It also provides an analysis of research conducted with first, second, and third graders in public and private schools of the Costa Rican Education System. Using questionnaires and observations of the dynamics of the classroom as measurement tools, this study contrasts teachers' expectations about their students' talent with the reality of the class environment, pointing out the existing abyss between teachers' beliefs and their professional practices.

Key words. Children from first to third level of primary school, talent, teachers' practices, educational reality, teachers' beliefs.

\section{Introducción}

En un afán de compartir conocimientos sobre el tema del talento en niños y niñas de I Ciclo de la Educación General Básica, se retoman en este artículo algunos de los datos encontrados, mediante una investigación realizada por Castro, Solís, Ureña y Villalobos (2007), la cual buscó contrastar, respecto al tema en cuestión, la perspectiva docente con las vivencias y observaciones de aulas, en instituciones educativas costarricenses.

La investigación de Castro et al. (2007) señala cómo, en el tema del talento, hay variabilidad de conceptualizaciones; no obstante, en la investigación dicha se asumió el concepto de la existencia de un talento en cada niño y niña, y se señaló la necesidad de que las instituciones educativas, desde esa perspectiva, logren responder a las necesidades de desarrollo integral de sus estudiantes.

Si la educación es un derecho de todos y todas, consideramos que la misma ha de buscar el desarrollo integral de los que la reciben; ha de ser una educación encaminada al fortalecimiento de habilidades, talentos y promotora de la construcción de conocimientos dentro de un ambiente sano y estimulante. Llevar a cabo este derecho implica respetar el principio de igualdad de oportunidades, y brindar las ayudas y recursos requeridos por el estudiantado, en función de sus características y necesidades individuales.

En este punto, quien ejerce la docencia adquiere un papel protagónico en la detección y atención de los talentos que poseen sus alumnos y alumnas, para brindarles una educación integral, que potencialice sus habilidades y capacidades. De igual manera debe conocer sus características y necesidades educativas, para ser capaces de responder a ellas con creatividad. Así, mediante la adaptación curricular, demuestra su capacidad para trabajar en función de la estimulación de los talentos de sus alumnos y alumnas.

En Costa Rica, a lo largo de los años, se han llevado a cabo investigaciones afines a la temática de este artículo. Dentro de estas, la de Beirute y De Bravo (1984), en la Universidad de Costa Rica, 
ha sido pionera. Tomó en cuenta el fomento de la creatividad en las niñas y niños talentosos, de esta manera, estableció que la creatividad es un aspecto que puede estimularse y promoverse; además, demostró que las capacidades creativas del niño(a) permanecen subdesarrolladas y pueden hasta atrofiarse, si el ambiente educativo no las estimula. Destacan las autoras que el principal aspecto para ser creativo, lo constituyó la metodología que se seguía en el aula, la presencia del ambiente liberador y el tipo de experiencias utilizadas.

Por otra parte, Alvarado (1993) promovió metodologías creativas para la enseñanza de la guitarra en III y IV ciclos de los colegios urbanos, mediante talleres con un "modelo curricular de voluntaria elección”, con una pedagogía de acción en la que imperó el interés y la motivación del educando. En ese sentido, brindó oportunidades para responder a los talentos o intereses de quienes escogieron esa opción.

En otra investigación, Cascante y Meléndez (1993) elaboraron una propuesta de orientación profesional, para la atención, en los colegios científicos costarricenses, de estudiantes talentosos, a partir de sus características en cuanto a interés y aptitudes. Utilizaron un referente de talento ligado a las notas de los estudiantes. A pesar de que hicieron referencia a diferentes áreas donde se puede manifestar el talento, se tomó en cuenta para efectos del trabajo, únicamente, el talento intelectual.

Dicha investigación corroboró la capacidad de los estudiantes y las estudiantes para resolver problemas, así como para realizar experimentos y lecturas, involucrando, en su quehacer personal, la perseverancia, sin dejar de lado características como la observación, la criticidad y el perfeccionismo. Se observó, además, que el estudiante talentoso se caracterizaba porque disfrutaba de la actividad intelectual, la cual se pudo canalizar en ese caso mediante la lectura. También se visualizó que, tanto lo social como lo deportivo, predominaban sobre lo artístico.

Cabe destacar que la mayoría de estudios realizados en Costa Rica y relacionados con la temática del talento se ha abordado desde una perspectiva intelectual, concepto considerado como una cualidad extraordinaria en una persona.

La investigación que más se acerca a los fines de este artículo es la de Chávez y Van Brown (1997), quienes abordaron el talento desde el área intelectual, aunque hacen énfasis en que todas las personas poseen algún tipo de talento, el cual debe ser desarrollado y potencializado. La definición de talento que las autoras aportan es la siguiente:

Un niño con talento, es aquel que da muestras de creatividad, originalidad, que muestra dominio de los materiales y capacidad para jugar con ellos, realiza técnicas cognoscitivas y constructivas con facilidad, que es innovador, trabaja en función de un tema y a partir de una realidad. (p. VII)

En su trabajo investigativo, dichas autoras aplicaron técnicas educacionales en escuelas públicas urbanas que tenían en sus aulas a niños y niñas talentos. Concluyen que poseían capacidad intelectual sobresaliente, la cual los diferenciaba del grupo. Al aplicar la prueba de Piers-Harris, descubrieron que estos niños y niñas mostraban buen comportamiento, bajos niveles de ansiedad, alto status escolar e intelectual y que eran felices; también encontraron diferencias entre el concepto de talento por parte del docente y las estrategias metodológicas utilizadas en el aula.

En un estudio de caso, Morales y Salas (2005) realizan un interesante análisis que retoma las condiciones familiares, socioeconómicas y contextuales de una familia y su hijo talentoso. Resaltan las autoras la importancia de que los padres se planteen ideales altos, así como que haya adecuados 
niveles comunicativos, a pesar de permanecer en condiciones socioeconómicas bajas. Agregan las autoras citando a Heward (1998) que los niños superdotados y talentosos son aquellos que:

Poseen capacidades demostradas o potenciales que implican un alto nivel de desempeño en tareas cognitivas, en tareas que requieren de una solución creativa y en áreas escolares, y que por ello requieren servicios y actividades diferentes a las que la escuela suele proporcionar. (p. 6)

Señalan las autoras que debe analizarse si el sistema educativo costarricense posee las condiciones para fortalecer y estimular a la niñez considerada talentosa y con el estudio se responden que, realmente, se requiere de capacitación, sensibilización y cooperación para poder atender de manera adecuada los talentos de niños y niñas.

Beirute (2008) plantea que en el Instituto Educativo Moderno (IEM) institución educativa de Costa Rica, se concibe el talento “(...) como la presencia de habilidades superiores en un niño o niña al compararla con sus pares en cualquier área (académica, social, deportiva, artística, tecnológica, ecológica...)" (J. 44). Señala la autora que esa capacidad superior no se circunscribe a valoraciones psicométricas, sino que es visualizada como producto del desempeño cotidiano del estudiante y la estudiante en el aula, paralelo a un seguimiento de su proceso de desarrollo socioemocional y de rendimiento en distintos escenarios.

Acota, además, que para fortalecer la identificación y el desarrollo de dichos talentos, la institución ha reforzado y diversificado la propuesta curricular y ha insertado una serie de proyectos, clubes, y talleres en diferentes áreas, dirigidos a toda la población estudiantil, donde el papel que juegan las familias es también prioritario.

Los estudios citados permiten demostrar la importancia que el tema del talento tiene en el ámbito educativo y se constituyen en ejemplos de cómo el concepto de talento ha sido abordado en diferentes investigaciones, desde una visión muy ligada al desempeño académico, hasta llegar a conceptualizaciones donde se asume la presencia de talentos en toda persona. Estas consideran otro tipo de variables para promover su atención, como bien pueden ser la familia, los procesos afectivos y comunicativos, la adecuación del curriculum, entre otros.

Por otra parte, la investigación realizada por Castro, et al. (2007), insumo para este artículo, se abocó a la profundización del tema del talento, con la perspectiva de que cada niño o niña del salón de clase posee uno o varios talentos, los cuales el docente y la docente debería de potencializar y ampliar al máximo, para el desenvolvimiento integral de la persona.

Por su parte, Agüero, Solano y Valdivia (2002) destacan la importancia de que tanto los padres, como los maestros, apliquen metodologías que estimulen el desarrollo de las potencialidades en el área de la inteligencia, habilidades, intereses y creatividad. Dejan claro que las características del talento en niños y niñas trascienden el cociente intelectual que les distingue, de modo que dejan ver la presencia de distintos talentos en ellos y ellas. Reconocen, también, que al hacer referencia al talento se han de destacar las habilidades y capacidades que todo ser humano posee. Justamente desde esta perspectiva se desarrolló la investigación que dio origen al presente artículo.

\section{Acercamiento teórico y metodológico}

Las instituciones educativas reciben estudiantes provenientes de poblaciones que, por su naturaleza, creencias, experiencias y estilos de vida, son diferentes, por lo cual, es también 
esperable y aceptable la existencia de diversidad de talentos en las aulas. En este sentido, es tarea de la educación capacitar para el ejercicio de la libertad y de la autonomía, lo que implica respeto para cada estudiante y para sus diversas manifestaciones, una de ellas, sus talentos. Si se parte de la idea de que cada persona posee uno o varios talentos, estos merecen ser reconocidos, respetados e incentivados en las diferentes dinámicas generadas en los procesos educativos.

La palabra talento proviene del latín "talemtum". Denomina una moneda antigua de los griegos. En el contexto social costarricense, se refiere a una actitud natural para realizar alguna cosa, entendimiento o inteligencia.

Actualmente se emplean otros términos como sinónimos de talento: excelencia, excepcional, superdotación, aprendizaje rápido, superior, brillante, dotado, sobredotado, superdotado, mejor dotado, superiormente dotado, entre otras.

En la bibliografía se encuentra una diversidad de términos que se relacionan con este concepto. Por ejemplo, superdotación, genio, precocidad, prodigio, talentos desaprovechados, talentos discapacitados.

Se produce una significativa confusión entre el término talento y superdotación, por ello, se considera importante hacer su respectiva distinción.

Al asumir la premisa de que todos los niños y niñas poseen uno o varios talentos y capacidades potenciales, en una o varias áreas de la actividad humana, apartándose de anteriores concepciones en las que, de manera restringida, se catalogaba como niño y niña con talento sólo a quienes poseen un alto cociente intelectual, cobra sentido la definición de Passow (citado por Benavides, Maz, Castro, y Blanco, 2004), quien plantea que “(...) el talento es la capacidad de un rendimiento superior en cualquier área de la conducta humana socialmente valiosa" (p. 32).

Se apela, así, al principio de igualdad y equidad, orientado desde la concepción de que todas las personas requieren oportunidades educativas que potencien sus talentos y destrezas, favoreciendo con ello, también, el desarrollo integral de su personalidad y el principio de respeto y valoración de toda habilidad o capacidad humana.

Para lograr lo anterior, se espera, entonces, que la labor realizada por el cuerpo docente se oriente a potencializar los talentos y se constituya en facilitador del proceso del desarrollo integral del alumno y alumna.

Sobresale así la necesidad de que el personal docente conozca las habilidades que poseen sus estudiantes, para que, en su función de mediador y facilitador de los procesos de aprendizaje, logre el desarrollo de los talentos de los niños y niñas.

De acuerdo con lo anterior, se pueden dividir los talentos en áreas que se relacionan con las características presentadas, las cuales se mencionan seguidamente:

\section{Tipos de talento}

Según Lorenzo (2006), el talento “(...) significa aptitud natural para hacer alguna cosa, entendimiento o inteligencia" (p. 173). Este concepto se ha empleado en una gran variedad de áreas y ha estado muy ligado, durante años, al concepto de superdotación, valorado mediante pruebas de inteligencia referidas a la medición de la capacidad de aprendizaje escolar de los alumnos (pruebas que se utilizan en la actualidad, para determinar el Coeficiente Intelectual (CI). No obstante, ha cobrado fuerza la conceptualización del talento como la capacidad o las capacidades específicas que posee una persona. 
Por su parte, autores y especialistas en la temática del talento han elaborado modelos y clasificaciones que tratan de acercarse a su comprensión. Así, por ejemplo, Mönks y Masson (citados por Lorenzo, 2006), clasificaron los diferentes conceptos de talento desarrollado a lo largo de la historia, en cuatro grupos: los orientados a rasgos o capacidades, los orientados a los componentes cognitivos, los orientados al logro y rendimiento, y los orientados a lo sociocultural y psicosocial. A continuación se ofrece una breve síntesis de las características fundamentales de cada una de estas clasificaciones, aportadas por Lorenzo (2006).

Orientación a los rasgos de la personalidad y a las capacidades. En esta clasificación se encuentran definiciones cuyo énfasis se basa en la inteligencia. Se señala, en este grupo, a quienes destacan por tener una inteligencia superior a la media; a quienes demuestran capacidades verbales superiores al promedio en pruebas de aptitud escolar. También, se menciona la presencia de habilidades superiores en áreas como: habilidad intelectual general, aptitud académica, pensamiento productivo o creativo, habilidades para el liderazgo, artes visuales o actuación, habilidades psicométricas, alto grado de pericia en un campo especial, o poseer un don innato o disposición natural hacia determinadas cosas por encima de la mayoría de personas, entre otras.

Como se puede observar, estas definiciones hacen referencia a la inteligencia vista como una capacidad general y abstracta, conceptualización que le brinda, a la persona poseedora de talentos, cualidades superiores a las de la media y dejan de lado el principio de igualdad.

Orientación a los componentes cognitivos. En este apartado se ubican aquellas definiciones referidas a reconocer los procesos, estructuras y estrategias cognitivas necesarias para llegar a la realización superior. No obstante, agregan los autores que se requiere de mayor verificación empírica que favorezca la comprensión del talento a partir del estudio de los procesos cognitivos utilizados en la resolución de diferentes tareas Mönks y Masson, 1993 (citados por Lorenzo, 2006).

Orientación al logro y al rendimiento. Se ubican en esta categoría los modelos que ponen en evidencia el talento como un aspecto en el que no solo interviene la inteligencia, sino también entran en juego factores tales como el logro y el rendimiento.

Dentro de esta clasificación se nota que las definiciones de talento se orientan a las aptitudes especiales de un niño o niña en una área específica; o una elevada inteligencia general por encima de la media.

Orientación a los elementos socioculturales y psicosociales. Dentro de esta clasificación se toman en cuenta aspectos como la familia, la escuela y la comunidad; así como la orientación económica, política y los valores culturales dominantes, como factores que intervienen en el desarrollo de los talentos de las personas.

En la misma línea de pensamiento, señalada con la clasificación anterior, se encuentra el planteamiento de Tannembaum, 1983 (Citado por Delgado, s. f.), quien define el talento de la siguiente manera:

En caso del talento, se ha de pensar en una serie de actividades que ocupen un área pequeña de la actividad humana general, donde la competencia y realización estarían por encima de la media de la población en las actividades mencionadas. Se podría considerar como una destreza cognitiva concreta. (p. 1) 
A partir de este concepto, el autor citado dividió el talento en 4 formas específicas: talentos escasos, talentos excedentes, talentos de cuota y talentos anómalos.

Sin embargo, esta clasificación recibió la crítica de varios autores. A partir de esta, la Oficina de Educación de los Estados Unidos, en 1972, propuso la división o estudio del talento en 6 áreas específicas, citadas por Delgado (s. f):

Habilidad intelectual general. Estas personas demuestran una alta capacidad de aprendizaje y un alto rendimiento escolar. Suelen poseer un alto sentido del humor, un pensamiento asociativo y divergente, con habilidad para generalizar, con actitud interrogante y crítica, junto con una alta perseverancia en las tareas. Debe valorarse también el tipo de juegos que realizan (basados más bien en reglas): pasatiempos, lecturas, profundidad de las soluciones y respuestas a los problemas, y una particular curiosidad por lo que les rodea.

Aptitud académica específica. Las personas que poseen este talento destacan en áreas determinadas del campo académico. Se dice que este aspecto es una derivación más específica del punto anterior. Estas personas pueden mantener intereses académicos muy enraizados, y obtener puntuaciones altas en ciertas áreas del ámbito escolar. Suelen reconocerse en este grupo el talento matemático; aunque sin dejar de lado el talento artístico o deportivo.

Pensamiento creativo y productivo. Las personas que manifiestan un pensamiento creativo y productivo destacan en la solución de problemas y en obras tangibles, independientemente del rendimiento que puedan obtener en un área determinada. Ponen en juego sus habilidades mediante la indagación de hipótesis, generando un elevado número de respuestas variadas en situaciones reales o hipotéticas, y relacionando ideas similares o inconexas.

Liderazgo. Quienes poseen este tipo de talento son personas respetadas por los demás, con carisma, con ganas de asumir responsabilidades en un número elevado de tareas y cumplir con ellas.

Habilidades en las artes visuales y representativas. En el ambiente social estas habilidades suelen ser juzgadas como "bohemias". Las personas que poseen este tipo de talento se manifiestan mediante conductas perceptivas, representaciones y ejecuciones artísticas.

Habilidad psicomotriz. Dentro de esta clasificación se encuentran personas que poseen habilidades artísticas, entendiéndose éstas como danza, baile, teatro, entre otras; así como el deporte y sus diversos aspectos. Dejan de lado las habilidades intelectuales.

Las clasificaciones destacadas en los apartados anteriores son muestra de la diversidad de definiciones acerca del talento. Por ello, se anota, en las líneas siguientes, la conceptualización que aporta la Asociación Costarricense para la Detección y Promoción del Talento, la cual enmarca el planteamiento con el que coinciden quienes escriben este artículo:

El talento es aquella capacidad que se presenta como significativa en cualquier persona y que la hace sobresalir dentro de su grupo. Puede representarse en cualquiera de los siguientes aspectos, o puede manifestarse por la combinación de algunos de ellos:

- capacidad intelectual

- aptitud sobresaliente en algún área

- capacidad para el liderazgo

- capacidad en el área motora

- habilidad especial en las artes gráficas, dramáticas, literarias, musicales

- capacidad creativa o productiva 
En algunos o algunas, esta manifestación es clara y abierta; pero en la mayoría de las personas es un potencial que puede ser estimulado y favorecido por las circunstancias del ambiente. No es exclusiva de ninguna condición social, cultural, raza o credo. (Lorenzo, 2006, p. 13)

De esta definición es importante restacar que el talento no se conceptualiza como una característica cognitiva, limitada a un estado del Coeficiente Intelectual que convierte a la persona en un ser sobresaliente al promedio; sino, por el contrario, el talento se asume como una capacidad presente en cualquier persona y la cual puede ser estimulada y favorecida por el ambiente.

Las distintas clasificaciones acerca del talento se constituyen en guías para la detección, estimulación y desarrollo de los mismos, no sin antes dejar de considerar también, la relación existente entre la inteligencia y el talento.

\section{Inteligencia y talento}

Personajes históricos importantes, como Aristóteles y Haywood (citados por Méndez (2005), han conceptualizado el término inteligencia, ampliamente relacionado con el talento. Señala la autora que Aristóteles definió la inteligencia como “(...) un rasgo único de la persona, el cual depende de la herencia" (p. 7), mientras que para Haywood era “(...) el resultado de la interacción de factores genéticos y de la experiencia del sujeto" (p. 8).

Es interesante notar que, para ambos pensadores, la inteligencia tiene que ver con la herencia del individuo, es decir, que para ser inteligente se necesita traerlo genéticamente; pero, también, se necesita experimentar en el camino de la vida para descubrirla y desarrollarla con el paso del tiempo.

Sin lugar a dudas, las conceptualizaciones anteriores, aunque se realizaron específicamente para el término inteligencia, se relacionan con el concepto de talento, pues también este es asociado con la herencia genética de las personas y con las experiencias estimulantes que reciben del medio, lo que les permite desarrollarlo.

De acuerdo con Soriano (1996), “(...) investigaciones sicológicas (...) han destacado que uno de los determinantes más poderosos del comportamiento humano es la percepción que la persona tiene de sí” ((I. 8). Por esta razón, si la persona se percibe con características como la inteligencia y la capacidad para hacer bien las cosas, estas virtudes se verán reflejadas en sus actitudes ya que, el ser humano siempre busca congruencia entre la visión de sí mismo y su actuar. Es aquí donde tiene cabida el proporcionar, por parte del Ministerio de Educación, universidades, directores, docentes, padres de familia, entre otros, un aprendizaje innovador que ponga un alto al desperdicio del potencial creativo humano. Este desperdicio podría ser la consecuencia de una educación que adopta una visión castradora, opresora, anticuada, aburrida, tradicionalista y que lleva a quien está dentro de sus caminos a la reproducción y memorización de conocimientos. Una educación que deja atrás el verdadero disfrute del saber, crecer y conocer, logrado mediante una actitud creativa por parte de los sujetos, en un ambiente estimulante para su desarrollo.

$Y$ es que se sigue pensando que el talento es un don que está presente únicamente en algunas personas privilegiadas. Por tanto, si se nota algún talento en alguno de los niños y niñas que, como educadores se tienen a cargo, no se le da especial atención, pues los demás no lo tienen. Por ello, vale la pena recordar que el talento va relacionado con distintas inteligencias, de modo que alguien puede destacar en música, dibujo, matemáticas, lengua, deportes, relaciones interpersonales, $\mathrm{O}$ cualquier otra área (Lapalma, 2005). 
Si asumimos que cada individuo posee uno o más talentos, los cuales se hace necesario descubrir y desarrollar como parte de la formación integral del estudiantado, es posible asumir también que el cuerpo docente puede jugar un papel importante con sus estudiantes, los cuales pasan parte de su tiempo en las instituciones educativas. Por ello, creemos que el proceso de enseñanza y aprendizaje ha de ir acompañado por la estimulación, la atención, la aceptación, el afecto, la alegría, el placer por el aprendizaje y el reconocimiento al logro, entre otros aspectos. Y en esto cobra importancia no solo el papel del maestro y la maestra, sino también el de la familia, la comunidad y los distintos contextos en los que se desenvuelven nuestros niños y niñas.

La investigación realizada por Castro et al. (2007), de cuyos resultados se formula el presente artículo, fue de carácter exploratoria descriptiva y se enmarcó dentro de un paradigma cualitativo, en el que se hizo una integración metodológica, mediante la utilización del cuestionario y la observación de clase como recurso prioritario. Tuvo como participantes a 64 docentes que laboraban en dos instituciones educativas de carácter público y dos escuelas de carácter privado, del circuito 03 de la provincia de San José, el circuito 02 y 04 de la provincia de Heredia y del circuito 01 de Puriscal, quienes, mediante la técnica del cuestionario, expresaron sus conceptos sobre talento, características de niños y niñas talentosos y talentosas, así como las estrategias utilizadas para fortalecer el talento en estudiantes de I ciclo de la Educación General Básica.

Se realizaron también 60 observaciones de la dinámica de aula en cuatro secciones de primer grado, cuatro de segundo y cuatro de tercero. Se utilizó la lista de cotejo como instrumento de observación. Se contabilizaron un total de 55 horas de observación de aula.

Los datos fueron transcritos textualmente y organizados por categorías de análisis, se integró, también, información mediante cuadros y figuras.

Se presentan, a continuación, algunos resultados que evidencian la brecha entre el concepto de talento de los docentes y las docentes y la realidad que se vive en las aulas del sistema educativo costarricense, particularizadas para los grupos participantes.

Al iniciar el análisis, es importante aclarar que, de los 64 docentes participantes en una primera fase, 55 fueron mujeres (86\%) y 9 hombres (14\%); de los cuales 28 tenían más de 40 años (44\%), 19 con edades entre 31 y 35 años (29\%), 14 de los participantes en edades entre los 26 y 30 años (22\%) y 3 eran menores de 25 años (5\%).

En cuanto al grado en el que laboran los docentes, se ubicaba un 34\% en tercer grado, $33 \%$ en primer grado y un $33 \%$ en segundo grado; sin embargo, cabe destacar que la mayoría, un 38\% de la población femenina entrevistada, atendía el primer grado y, en dicho grado inicial, no laboraban varones.

En cuanto al nivel académico de formación, el $51 \%$ de los docentes poseían el grado de bachillerato, 39\% licenciatura, $6 \%$ maestría y 2\% diplomado. Otro 2\% se ubicaba en la categoría de aspirante en educación. En términos generales, los participantes tenían un nivel alto de formación.

De los 64 participantes, el 50\% poseía experiencia en el campo docente que abarcaba entre los 6 a 15 años de labor, 30\% de los docentes contaba con más de 15 años de servicio y un $20 \%$ tenía menos de 5 años de experiencia.

Al indagar sobre la cantidad de alumnos que atienden en I Ciclo, se obtiene que un $30 \%$ atiende a grupos constituidos por una población entre los 26 a 30 alumnos, 23\% trabajan con grupos entre 31 a 35 alumnos, $17 \%$ con grupos de 36 a 40 alumnos, 8\% de 21 a 25 alumnos, 8\% de 16 a 20 alumnos, $6 \%$ de 10 a 15 alumnos, $5 \%$ con más de 40 alumnos y un $3 \%$ con grupos de menos de 10 alumnos. Se puede observar que la mayoría atendía grupos numerosos (con más de 26 estudiantes), lo cual, como es esperable, provoca mayor dificultad para la atención personalizada en función de las necesidades y talentos del grupo estudiantil. 
La observación de aula como recurso para la recolección de datos permite destacar algunos resultados importantes, para contrastar la opinión de los docentes y las docentes, manifiesta en el cuestionario y la realidad de aula. Se destacan a continuación, mediante figuras, algunos de los datos que pueden ilustrar de manera más precisa este aspecto:

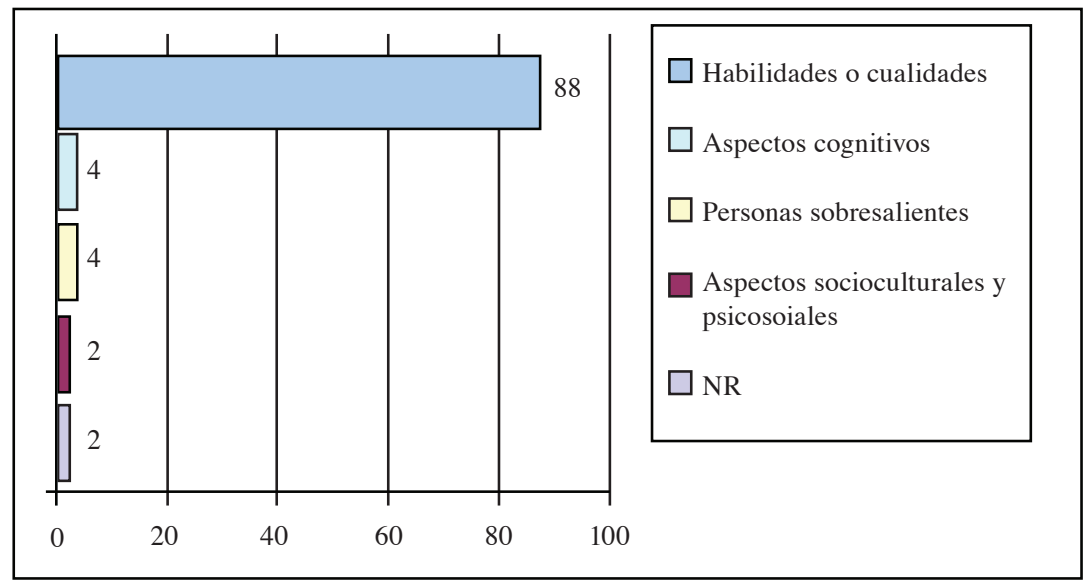

Figura 1. Concepto de talento expresado por los docentes y las docentes de I Ciclo

La Figura 1 permite observar que de los 64 docentes a quienes se les aplicó el cuestionario sobre su perspectiva acerca del talento, el 88\% lo conceptualizó como la manifestación de los rasgos y capacidades de la persona. El $4 \%$ se refirió al talento como un componente cognitivo, o como una característica única de personas sobresalientes y $2 \%$ lo definió como un elemento sociocultural y psicosocial; por otro lado, un $2 \%$ no respondió la pregunta.

De acuerdo con el concepto brindado por las docentes y los docentes participantes, se visualiza que la mayoría posee una perspectiva de talento que coincide con la planteada en la investigación, como un talento en cada niño y niña. Una minoría lo conceptualiza como un aspecto referido al desarrollo cognitivo. En ese sentido, existe coincidencia en los datos encontrados con los planteamientos que acerca del talento se ofrecen en la bibliografía. Es importante resaltar que el $2 \%$ de los participantes no respondió a este cuestionamiento, lo que puede indicar desconocimiento acerca del tema o, simplemente, no tener deseos de responder.

Cuando se indagó acerca de la presencia o no de talentos en sus alumnos y alumnas, fue muy interesante notar que una amplia mayoría indica que denotan talentos en sus estudiantes. Expresarse en términos diferentes no sería algo esperable dentro del discurso del "deber ser" que pesa mucho dentro del ámbito docente. La figura siguiente muestra el comportamiento de las respuestas en el sentido señalado: 


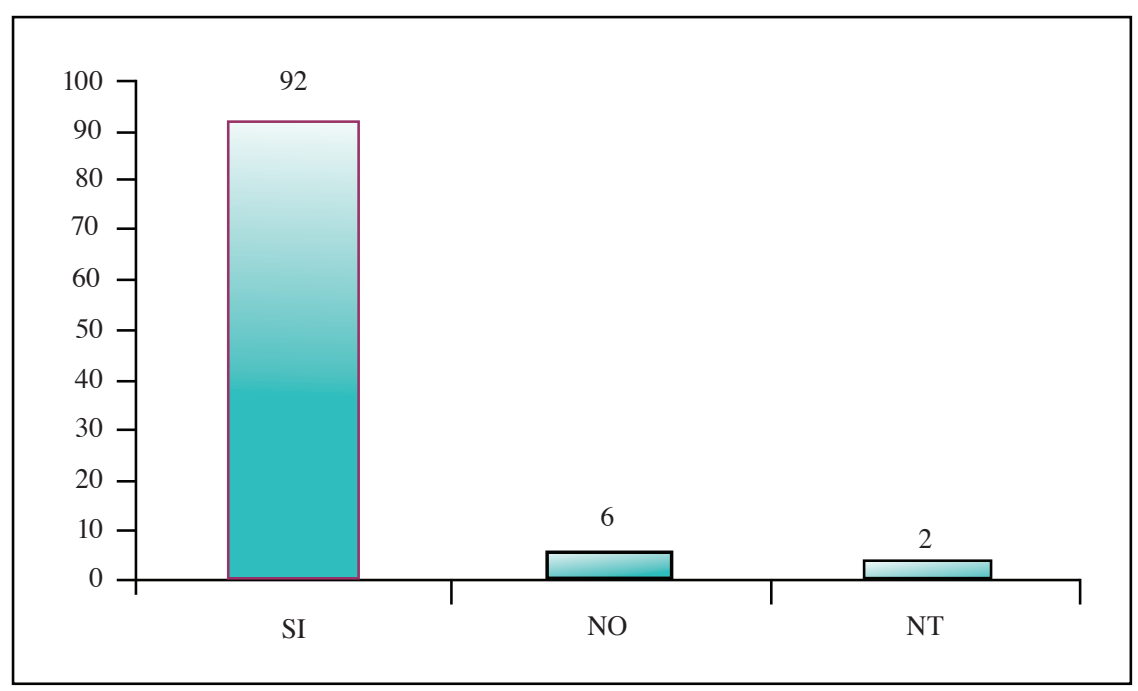

Figura 2. Opinión de los docentes y las docentes de I Ciclo, acerca de la existencia de talentos en sus alumnos y alumnas.

Es notorio como en la Figura 2 se indica que un $6 \%$ de las docentes participantes, abiertamente señalaron que no hay talento en sus alumnos y alumnas, y un $2 \%$ no da respuesta a lo planteado, aspecto que, de igual manera, puede interpretarse como desgano por atender el tema o desconocimiento acerca del mismo; ambos señalamientos motivo de preocupación desde los objetivos de la práctica profesional en el campo educativo.

Tal como lo muestra la Figura 2, es interesante señalar que, a pesar de que las docentes manifiestan que en sus alumnos y alumnas hay talentos, cuando se les cuestiona acerca de la cantidad de alumnos y alumnas talentosas que tienen en sus aulas, citan a muy pocos estudiantes. Los datos siguientes lo demuestran:

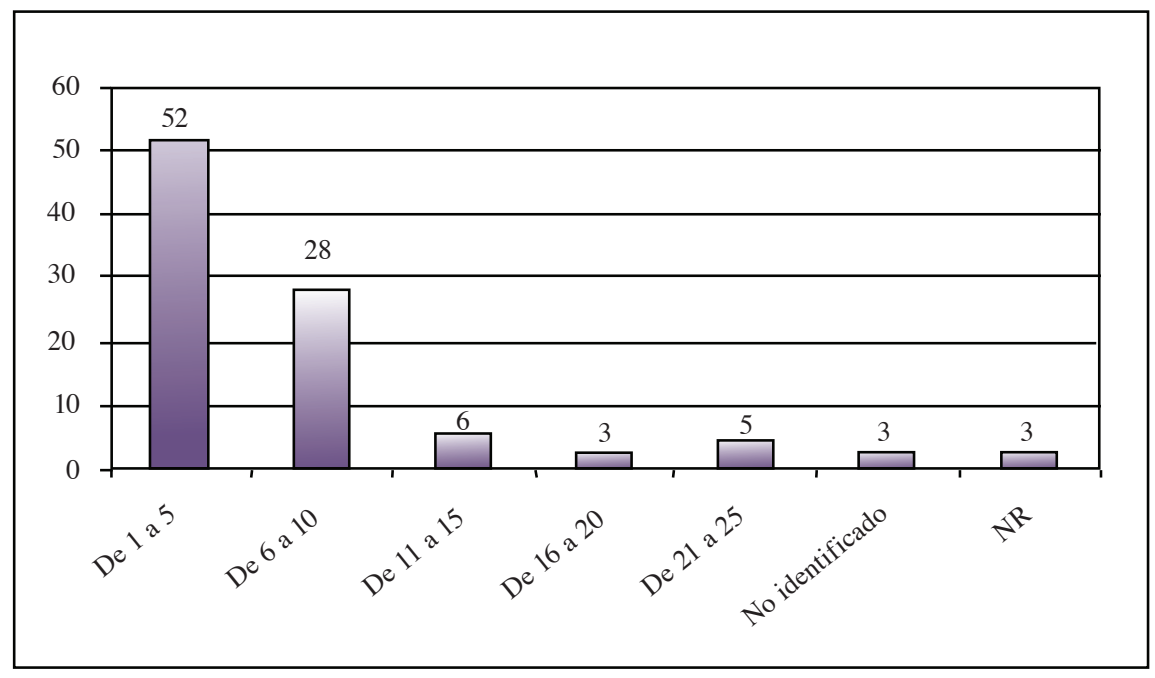

Figura 3. Número de alumnos y alumnas talentosos que tienen en el aula, según opinión de los docentes y las docentes. 
La Figura 3, muestra que; no obstante lo dicho acerca del talento y la identificación de este en sus alumnos y alumnas, solo un 53\% de los docentes y las docentes de I Ciclo identifican de 1 a 5 estudiantes con talento en su aula. Es preocupante que al día de hoy, cuando el avance teórico hace ver la presencia de uno o varios talentos en cada persona, el cuerpo docente de I Ciclo señale, prioritariamente, que el talento lo presentan muy pocos de sus estudiantes.

Igualmente preocupante es el caso de los profesionales y las profesionales que guardaron silencio ante la pregunta formulada (3\%) y quienes abiertamente señalan que no han identificado talentos en sus alumnos y alumnas (5\%).

Lo dicho quizá tenga una fuerte relación con los datos siguientes, en donde, de acuerdo con la opinión docente, se indican cuáles son las áreas en las que identifican los talentos de sus alumnos y alumnas, y cómo se prioriza en lo artístico, razón que quizá condicione, de alguna manera, lo que indican acerca del número de niños y niñas con talento en sus aulas.

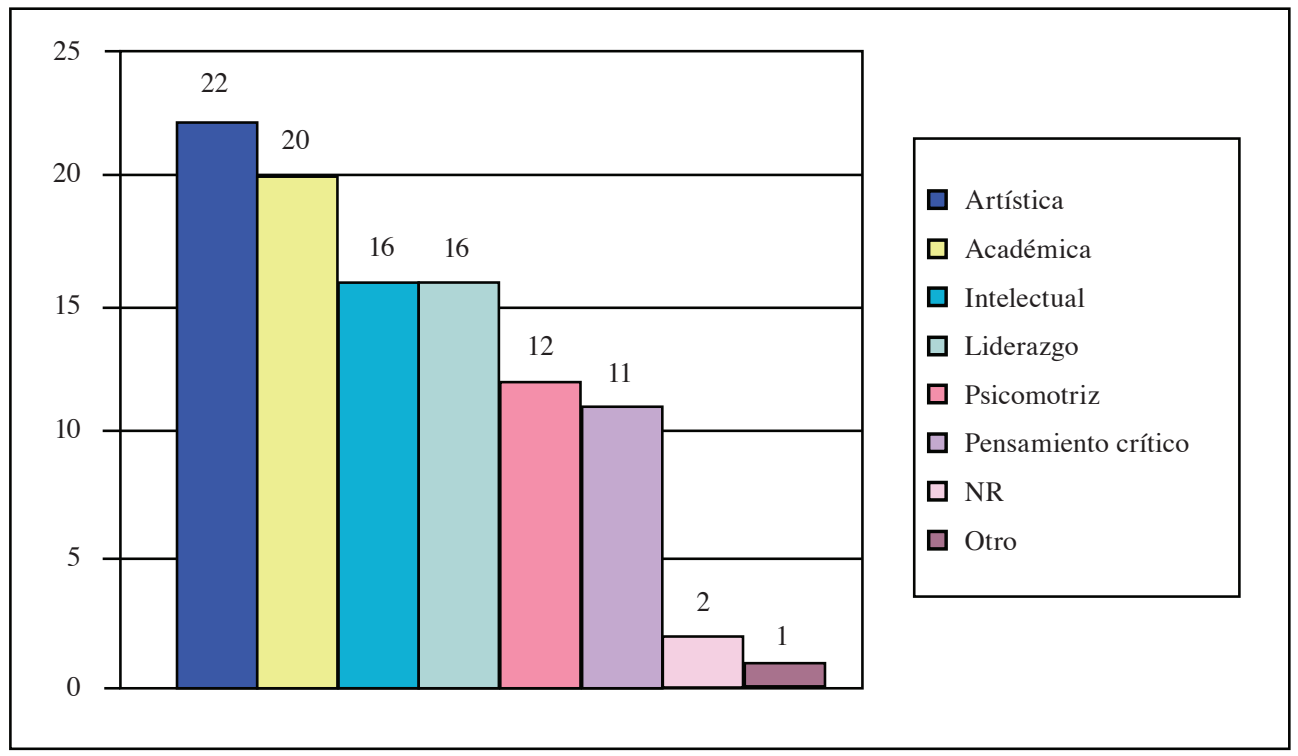

Figura 4. Áreas en las que los docentes y las docentes de I Ciclo ubican los talentos de sus alumnos y alumnas.

La figura 4 muestra cómo los docentes de I Ciclo relacionaron el talento con el cociente intelectual y el desempeño académico y artístico, por lo cual las áreas que, prioritariamente, son mencionadas como talento en el estudiantado son, justamente, la artística (22\%) y la académica (20\%). Por otra parte y muy relacionadas con el cociente intelectual o habilidades cognitivas, están las áreas intelectual y de liderazgo, ambas mencionadas por un $16 \%$ de los docentes y las docentes de I Ciclo participantes de la investigación.

Cuando se indaga con estos profesionales acerca de las estrategias utilizadas para atender el talento de sus estudiantes, es interesante notar, a partir de las 60 observaciones de aula realizadas, que hay un comportamiento muy desigual en el uso de estrategias en relación con lo que mediante el cuestionario expresaron, notándose un abismo entre lo que se dice y lo que realmente sucede en las aulas costarricenses observadas. 
Pareciera que en el discurso del "deber ser" se hace referencia a estrategias para fortalecer los talentos estudiantiles; no obstante, mediante observaciones de la práctica profesional se constata la ausencia de dichas estrategias, de modo que hay un evidente contraste entre el ser y el deber ser de la práctica docente.

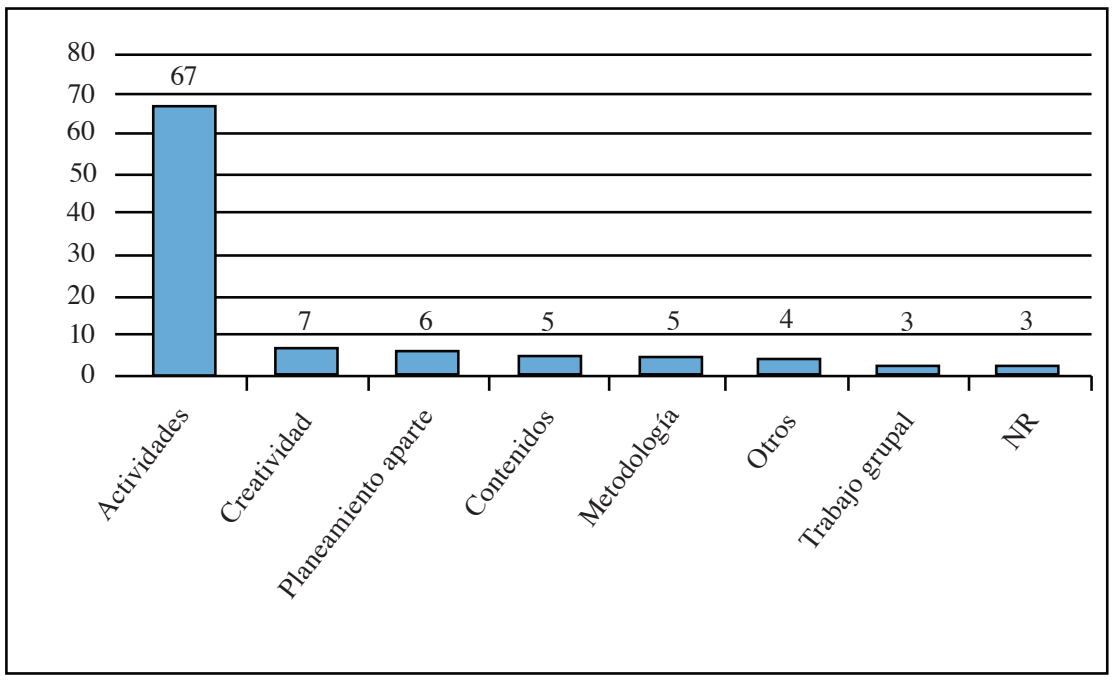

Figura 5. Estrategias utilizadas por los docentes y las docentes de I Ciclo, para estimular el talento de alumnos y alumnas, según observaciones de aula.

La Figura 5 denota que, en un $67 \%$ de las observaciones de aula, se pudo constatar que los docentes y las docentes de I Ciclo utilizaban diversas actividades de trabajo como: "actos cívicos", "actividades deportivas", entre otras. No obstante, es notorio cómo la diversidad metodológica y el trabajo en grupos, dentro de otras alternativas, son las menos utilizadas; a pesar de que los estudios por años ha sido claros en señalar que las actividades lúdicas, el aprendizaje compartido y la diversidad metodológica se constituyen en elementos claves para el aprendizaje. Factor, que a criterio personal, ha de ser seriamente evaluado por los profesionales y las profesionales en el campo educativo.

Quien educa adquiere la función de facilitador y motivador de los talentos descubiertos en sus estudiantes, por lo que su labor es esencial para atender, mediante diferentes estrategias, los diversos talentos que poseen sus discentes; dejando de lado las prácticas tradicionales basadas, en su mayoría, en el aprovechamiento de los talentos estudiantiles en los actos cívicos, competencias o concursos.

La siguiente figura representa el tipo de talento que es frecuentemente reforzado por los docentes y las docentes en el trabajo de aula, aspecto que fue registrado mediante las observaciones realizadas en las aulas: 


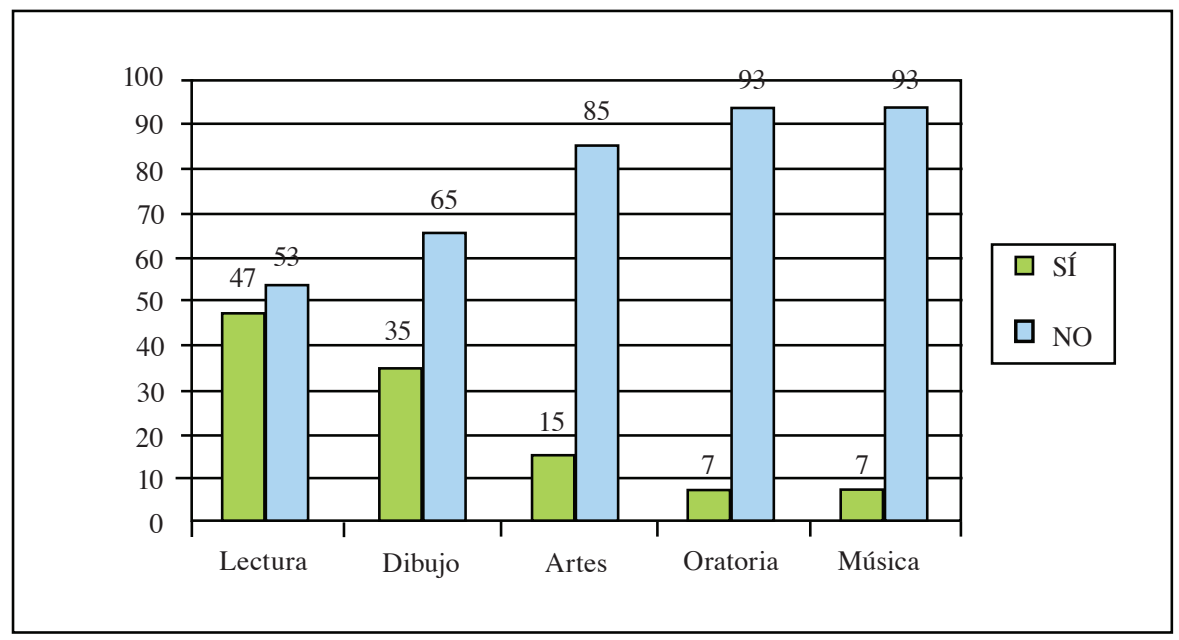

Figura 6. Tipos de talento desarrollados en el aula.

Es notorio cómo se suele priorizar el talento que más directamente se relaciona con capacidad cognitiva y éxito escolar, a saber: lectura y dibujo. No obstante, áreas importantes como arte, oratoria y música, que también son altamente relacionadas con el talento, no son reforzadas mediante el desarrollo de actividades en el aula escolar.

Los docentes y las docentes, en el trabajo de aula, dejan de lado las concepciones de talento referidas a su presencia en cada persona. Por tanto, en sus estrategias de trabajo, están ausentes los elementos que considerarían la atención del talento dentro del planeamiento de aula, por lo que es posible señalar que existe un divorcio entre las conceptualizaciones que acerca del talento plantean y lo que realmente ponen en práctica en su trabajo de aula.

Vale la pena rescatar aquí el planteamiento de Beirute (2008), quien señala la necesidad de que en el trabajo de aula los docentes estimulen los talentos, aspecto que también es planteado de manera explícita por Morales y Salas (2005).

\section{Reflexiones finales}

Los planteamientos de Morales y Salas (2005) y Beirute (2008) hacen notoria la importancia de la institución educativa en la búsqueda de alternativas y el compromiso expreso para detectar, promover y fortalecer los talentos en niños y niñas.

Se vislumbra, en las diferentes investigaciones llevadas a cabo en el ámbito nacional, la necesidad existente de que, dentro del sistema educativo costarricense, se atienda a niños y niñas y se les brinden las oportunidades para el desarrollo de sus talentos, donde puedan plantearse preguntas, explorar, investigar y buscar respuestas alternativas; donde fluya la comunicación, y el currículo realmente responda sus necesidades e intereses. Aspectos todos plenamente compartidos en nuestro planteamiento.

Sin embargo, contrastando los requerimientos para la atención de niños y niñas talentosas, los criterios expresados por docentes que imparten I Ciclo y la realidad que se vive en los salones de clase, hemos encontrado una divergencia entre esos elementos. Se enumeran a continuación las principales conclusiones que se destacan a partir de los datos recopilados y expuestos anteriormente: 
- La mayoría de los docentes y las docentes posee un concepto teórico de talento, basado en las diferentes habilidades y capacidades que tienen las personas, visualizan el talento como capacidades en las diferentes áreas de la conducta humana.

- En la práctica cotidiana, en el desarrollo de las lecciones, no se toma en cuenta lo que expresan sobre el talento, ya que los docentes y las docentes trabajan con un principio de homogeneidad y uniformidad de sus discentes.

- Aunque los docentes y las docentes de I Ciclo argumentan que el talento se haya presente en áreas específicas de la conducta humana, en su práctica profesional las áreas mayoritariamente estimuladas son la lectura y el dibujo, y le restan importancia a artes, oratoria y música, habilidades que también permiten el desarrollo integral de los estudiantes y las estudiantes.

- La atención que el grupo de docentes brinda a los talentos de sus estudiantes se orienta, primordialmente, a la estimulación, participación y realización de actividades denominadas como tradicionales, tales como actos cívicos y festivales deportivos. Esto demuestra que no se realizan actividades variadas para el estímulo de los talentos en especial.

- Es importante rescatar que los grupos observados están conformados por entre 26 y 40 alumnos y alumnas, lo que de una u otra manera, puede significar una limitante para la atención de los talentos estudiantiles; pero, a pesar de ello, siempre mediante metodologías diversas y creativas y utilizando la estimulación, puede ser posible el logro del desarrollo del potencial de los alumnos y alumnas.

- De acuerdo con las observaciones realizadas dentro del ambiente de aula, se visualizó la promoción de estrategias de aprendizaje individualizadas, ya que, en su mayoría, tienen los pupitres ubicados en filas individuales como estrategia para el manejo de límites, con lo cual se deja de lado el proceso socializador y comunicativo que se debe promover en los salones de clase, para que los estudiantes y las estudiantes compartan entre sí sus habilidades y capacidades y, de esta manera, fortalezcan sus talentos.

- Las estrategias puestas en práctica para el desarrollo de las lecciones por parte delgrupo de docentes observado, se enfocaron en el desarrollo de los contenidos propuestos por el Ministerio de Educación Pública Costarricense, sin considerar otras estrategias para la estimulación de sus estudiantes, lo que provoca que, en muchas ocasiones, se subestimen sus talentos.

\section{Referencias bibliográficas}

Agüero, H., Solano G y Valdivia, S. (2002). Propuesta Didáctica para Estimular el Desarrollo de las Inteligencias en la Enseñanza de la Unidad "La Célula" del Programa de Ciencias de Noveno Año. Tesis inédita de Licenciatura no publicada. Universidad Nacional, Heredia, Costa Rica.

Alvarado, A. (1993). Talleres de creatividad, talento y recreación: la enseñanza de la guitarra como una opción metodológica en los colegios urbanos del país. Tesis inédita de Licenciatura no publicada, Universidad Nacional, Heredia, Costa Rica.

Beirute, L. y de Bravo, O. (1984). El niño talentoso en Costa Rica: La adecuación de un currículum para el fomento de su creatividad en el aula regular. Tesis inédita de Licenciatura no publicada, Universidad de Costa Rica, San José, Costa Rica: Instituto Educativo Moderno. 
Beirute, L. (2008). El IEM en Costa Rica: una experiencia enriquecedora de atención al talento en el aula regular. Ponencia presentada en el I Congreso internacional incursiones educativas en superdotación y talentos sobresalientes. San José, Costa Rica: UNED. Disponible en http:// www.iemonline.org/talento.pdf

Benavides, M., Maz, A., Castro, E. y Blanco, R. (2004). La educación de niños con talento en Iberoamérica. Santiago, Chile: UNESCO. Disponible en: http://unesdoc.unesco.org/ images/0013/001391/139179s.pdf

Cascante, V. y Meléndez, V. (1993). Los estudiantes talentosos de los colegios científicos costarricenses. Propuesta para su atención en la reorientación profesional. Tesis inédita de Licenciatura no publicada, Universidad Nacional, Heredia, Costa Rica.

Castro, D., Solís, J., Ureña, K. y Villalobos, B. (2007). Talento en niños y niñas del I ciclo de la Educación General Básica: la perspectiva docente. Tesis inédita de Licenciatura de Licenciatura no publicada. Heredia: Universidad Nacional.

Chávez, H. y Van Browne, M. (1997). Técnicas educacionales para los (as) docentes de escuelas públicas urbanas que tienen en sus aulas a niños (as) talentosos (as). Tesis inédita de Licenciatura no publicada, Universidad Nacional, Heredia, Costa Rica.

Delgado, R. (s. f.). El talento. Recuperado de http://www.terra.es/personal/asstib/talento.htm

Lapalma, F. (2005). Las inteligencias múltiples y el talento. En línea: Recuperado de http://www. rieoei.org/deloslectores/1100Lapalma.pdf

Lorenzo, R. (2006, Enero-Marzo). ¿A que se le denomina talento? Estado del arte acerca de su conceptualización. Intangible Capital, $\mathrm{N}^{\circ}$ 11, Vol. 2, 72-163. Recuperado http://www. intangiblecapital.org/index.php/ic/article/viewFile/47/49

Méndez, Z. (2005). Aprendizaje y cognición. San José, Costa Rica: Editorial Universidad Estatal a Distancia.

Morales, M. E. y Salas, N. (Enero-Junio, 2005). Cruzando fronteras, un caso de talento desde la escasez: una experiencia comunicacional con un niño "gifted" (Superdotado y Talentoso). Revista Latina de Comunicación Social. Vol. 8, Número 059. Recuperado de http://redalyc. uaemex.mx/redalyc/pdf/819/81985909.pdf

Soriano, E. (1996). La escuela y el desarrollo del talento creativo. Un desafío para las Américas. Portal Educativo de las Américas. Colección: La Educación. Número (123-125) I, III. Disponible en http://www.educoas.org/portal/bdigital/contenido/laeduca/laeduca_123125/articulo9/index.aspx 